\title{
Introduction : réhabilitation, reconversion et renouvellement des espaces industriels et urbains dégradés
}

\section{Michel Deshaies}

\section{(2) OpenEdition}

Journals

Édition électronique

URL : http://journals.openedition.org/rge/1384

DOI : $10.4000 /$ rge.1384

ISSN : $2108-6478$

Éditeur

Association des géographes de l'Est

Édition imprimée

Date de publication : 1 juin 2006

ISSN : 0035-3213

\section{Référence électronique}

Michel Deshaies, «Introduction : réhabilitation, reconversion et renouvellement des espaces

industriels et urbains dégradés », Revue Géographique de l'Est [En ligne], vol. 46 / 3-4 | 2006, mis en

ligne le 18 décembre 2009, consulté le 21 septembre 2020. URL : http://journals.openedition.org/rge/ 1384 ; DOI : https://doi.org/10.4000/rge.1384

Ce document a été généré automatiquement le 21 septembre 2020.

Tous droits réservés 


\title{
Introduction : réhabilitation, reconversion et renouvellement des espaces industriels et urbains dégradés
}

\author{
Michel Deshaies
}

1 L'espace géographique évolue, souvent lentement, parfois rapidement, voire brutalement, en particulier lorsqu'un système économique se substitue à un autre qui a structuré l'espace suivant une logique différente. C'est ce qui s'est produit, au cours des trente dernières années, dans de nombreuses régions d'Europe et d'Amérique du Nord, où le système industriel qui dominait depuis le XIX ${ }^{e}$ siècle, a subi de profondes transformations, voire s'est effondré pour céder la place au système que l'on qualifie en conséquence de post-industriel. Si le passage d'un système à l'autre a été relativement précoce et progressif en Europe occidentale, il a été par contre tardif et brutal en Europe centrale et orientale où le processus a suivi directement l'effondrement des régimes communistes en 1989.

2 Les conséquences spatiales de ces transformations sont très visibles dans les villes, notamment celles des vieilles régions industrielles où se sont multipliées les friches, espaces en déshérence, ayant en quelque sorte perdu leur fonction et pour lesquelles il a été le plus souvent difficile de retrouver un usage dans le cadre du nouveau système économique : vieilles usines délabrées, anciens carreaux de mines, ou vastes friches stériles lorsque les infrastructures et les bâtiments ont été rasés, constituent le produit de ces mutations et traduisent l'inadaptation du contenant (le bâti) au contenu (les activités économiques). Or, le devenir de ces espaces déchus et en conséquence dégradés, structurés et façonnés par un système industriel en déclin, ou ayant même totalement disparu, constitue un enjeu majeur pour l'avenir des villes et des régions concernées. Comme le souligne Guy Baudelle (1995) dans sa thèse sur le bassin houiller du Nord-Pas-de-Calais, "le traitement de ces paysages fossiles, stigmates de l'effondrement d'un système, s'avère d'autant plus indispensable que ceux-ci présentent des 
traits dissuasifs pour les investisseurs. Ces formes peu engageantes, en particulier les friches industrielles et urbaines, les décharges, les espaces dégradés et tous les délaissés, pour reprendre un terme technique, compromettent l'installation de nouvelles activités en conférant à ces lieux une image négative et répulsive ».

Réhabiliter ces espaces dégradés signifie d'abord qu'il faut les adapter au nouveau système économique et leur restituer un nouvel usage en les réaménageant, autrement dit les reconvertir. Mais les possibilités de reconversion dépendent d'abord de l'évolution économique régionale, de la localisation des friches, de la nature des projets et des acteurs qui réalisent la réhabilitation : opérateur privé ou institution publique, État ou collectivité territoriale. Pour permettre le «recyclage » des espaces dégradés laissés en friche par le déclin de l'ancien système en y implantant de nouvelles activités, il est toujours nécessaire de leur faire subir des opérations chirurgicales de plus ou moins grande ampleur, destinées à permettre leur réinsertion dans le nouveau système. Cela consiste à détruire une partie des anciennes infrastructures et des anciens bâtiments non réutilisables et à réaliser de nouvelles infrastructures destinées à accueillir les activités de la reconversion. En pratique, beaucoup d'opérations de réhabilitation aboutissent à faire table rase du passé; mais il est parfois possible de conserver certains éléments à valeur patrimoniale pour les réaffecter à de nouveaux usages, ou plus simplement pour donner une identité au site d'accueil de nouvelles activités : une ancienne usine à l'architecture remarquable comme à Roubaix, les restes d'un haut fourneau comme sur le site de Belval, à Esch-sur-Alzette, dans l'ancien bassin ferrifère luxembourgeois, ou encore un chevalement de mine comme dans la Sarre, ou à Faulquemont, dans l'ancien bassin houiller lorrain. On peut en effet exploiter le contenu patrimonial de ces espaces, en considérant que "l'avenir a besoin de racines » et que pour éviter la banalisation de l'espace, il convient de s'appuyer sur l'héritage historique, constituant en quelque sorte l'image de marque du site et un gage de qualité pour l'accueil des nouvelles activités. Le renouveau s'appuie alors sur un passé revalorisé et pour ainsi dire recyclé.

4 Cependant, si la crise qui marque le passage d'un système économique à l'autre est particulièrement brutale et profonde, se traduisant par un effondrement économique et une chute démographique importante, il est difficile de trouver de nouvelles affectations aux espaces en friche qui risquent alors de se pérenniser. Dans les régions les plus gravement affectées, c'est non seulement l'extension considérable des anciens espaces industriels en friche qui reflète l'ampleur de la crise, mais aussi l'ensemble de l'espace urbain où se multiplient les logements vides, ou en voie de dégradation. L'ampleur du problème dépasse alors largement la simple question de la reconversion et de la réhabilitation de friches industrielles, puisqu'il s'agit de rénover et de remodeler l'ensemble de l'espace urbain et en définitive de redéfinir la ville dont le cadre même se révèle complètement inadapté au nouveau système économique. Il s'agit alors, par des opérations multiples de rénovation ou de réhabilitation, de "régénérer» (Chaline, 1999) le cadre urbain hérité afin de créer les conditions d'un « renouvellement urbain » qui doit conduire à un changement d'image de la ville.

5 Les articles des géographes rassemblés dans ce numéro de la RGE, apportent des éclairages variés et complémentaires, à cette importante question de la réhabilitation et de la reconversion des espaces urbains et industriels dégradés qui sont analysés selon trois optiques inégalement présentes dans les différentes catégories d'espaces étudiés: les friches des bassins miniers, les espaces industrialo-urbains, les grands 
ensembles d'habitat collectif et les friches aéroportuaires militaires. Ces différentes optiques correspondent en fait plus ou moins, aux étapes successives du processus de « recyclage » des espaces dégradés qui, dans l'idéal, se déroule en trois phases souvent partiellement simultanées: requalification paysagère, réintégration dans la ville et reconversion économique. L'optique la plus largement présente est celle de la requalification paysagère, préalable indispensable à la reconversion. Dans les bassins miniers en priorité, mais aussi sur les espaces industrialo-urbains et dans les grands ensembles, on a engagé de lourdes opérations de chirurgie esthétique destinées à changer, au sens propre du terme, l'image de ces espaces. Cette requalification paysagère qui, en Lorraine, est assurée essentiellement par l'EPFL (Établissement public foncier Lorraine), constitue l'étape préliminaire d'un processus plus global d'aménagement urbain, visant à réintégrer dans la ville, ces espaces dégradés qui peuvent dès lors être de nouveau le support d'activités économiques nouvelles de reconversion.

6 Le numéro s'ouvre assez naturellement par trois articles consacrés à des bassins miniers qui constituent incontestablement les principaux gisements d'espaces industriels et urbains dégradés. En raison d'évolutions économiques et démographiques particulièrement négatives, mais aussi des spécificités de ces espaces, la réhabilitation, et à terme la reconversion, s'y déroule avec une lenteur particulière. En effet, le système mis en place autour des exploitations minières et des industries qui s'y sont développées se caractérise par sa rigidité et son incapacité à évoluer de l'intérieur sans subir une rupture majeure. Cette "rupture créatrice" (Holz, 1987), à l'origine du développement de nouvelles activités économiques susceptibles de se substituer aux activités traditionnelles, ne se produit le plus souvent qu'avec un certain retard par rapport à la crise affectant les piliers économiques traditionnels. Aussi, ce n'est parfois qu'après la disparition pratiquement totale des activités anciennes que se développe le processus de reconversion, permettant la réutilisation des espaces industriels dégradés, qui peuvent alors subsister longtemps dans les paysages.

7 À partir de l'exemple du bassin sidérurgique de Longwy, Colette RenardGrandmontagne montre ainsi que la lenteur de la reconversion s'explique par le temps considérable qui a été nécessaire pour que l'on fasse en quelque sorte le deuil de l'industrie. Après une quinzaine d'années de tentatives de reconversion industrielle pilotées par les institutions nationales et européennes, on s'est orienté depuis la fin des années quatre-vingt-dix vers une reconversion par le développement des activités commerciales qui a correspondu à l'engagement des collectivités territoriales. Cette nouvelle orientation a été en préparée par la requalification paysagère qui a abouti à faire table rase de presque tous les héritages industriels qui occupaient le fond de la vallée de la Chiers largement « renaturé ».

8 C'est aussi avec lenteur, mais pour d'autres raisons, que s'opèrent la requalification et la reconquête des héritages industriels et miniers du bassin houiller lorrain que nous présente Juliette Ripp. L'exploitation charbonnière a certes décliné depuis longtemps, mais s'est terminée beaucoup plus récemment que celle du bassin ferrifère et se poursuit d'ailleurs encore côté sarrois. En conséquence, seul le retrait progressif des charbonnages permet aux collectivités territoriales de récupérer les friches minières pour les valoriser, du moins une fois levées les servitudes minières. Contrairement au bassin ferrifère lorrain, où l'exploitation minière a laissé des héritages relativement 
modestes, l'exploitation houillère a marqué profondément les paysages, où les terrils et les immenses carrières occupent une place conséquente au sein d'une grande agglomération transfrontalière. C'est d'ailleurs l'importance de ces héritages, concentrés le long de la frontière avec l'Allemagne, qui rend d'autant plus nécessaire la réhabilitation de ces espaces dégradés dans le cadre de la coopération transfrontalière, structurée dans le « Parc de Développement de la Vallée de la Rosselle ».

9 Si la réhabilitation et souvent aussi la reconversion sont déjà largement amorcées dans les bassins miniers d'Europe occidentale, il en va tout autrement dans ceux des anciens pays communistes d'Europe centrale et orientale dont le déclin est postérieur à 1989. Corina Calugaru nous présente ainsi l'exemple du bassin de lignite d'Olténie en Roumanie. Il s'agit d'une exploitation à ciel ouvert à grande échelle, qui s'est développée récemment, à partir des années cinquante, et a profondément transformé les paysages initialement ruraux de la région. Comme dans tous les "pays de l'Est », la rupture politique de 1989 a entrainé de profonds changements économiques, qui se sont traduits par une chute de la production de lignite de près de $50 \%$, réduisant d'autant l'impact de l'exploitation minière et multipliant aussi les surfaces à réhabiliter. L'autre conséquence essentielle est le changement de la législation encadrant la réhabilitation. Alors que celle-ci était un objectif secondaire, largement négligé au temps du régime communiste, faute notamment de moyens financiers suffisants, elle est devenue une obligation pour les sociétés minières qu'elles doivent garantir par des dépôts bancaires. Mais en attendant, c'est l'État qui doit prendre en charge la réhabilitation des friches minières héritées de la période du régime communiste.

Les problèmes que posent la multitude et l'extension spatiale des espaces dégradés dans les bassins miniers peuvent se retrouver aussi, à une échelle moindre, dans des villes a priori moins marquées par l'industrie. C'est ce que montre Simon Edelblutte à partir de l'exemple du quartier Rives de Meurthe dans l'agglomération de Nancy. Il s'agit ici d'un quartier fortement marqué par le développement de l'industrie le long de la voie d'eau, à partir de la fin du XIX ${ }^{e}$ siècle et entré dans la spirale du déclin lié aux mutations économiques des années soixante-dix et quatre-vingt qui ont multiplié les friches. Mais la principale originalité du quartier est sa localisation à proximité du centre historique, notamment de la place Stanislas. Aussi, la réhabilitation du quartier, mise en œuvre par les autorités locales à partir des années quatre-vingt, est allée bien au-delà d'une simple opération de requalification et de reconversion. Le renouveau du quartier a conduit à une extension de l'espace péricentral de la ville sur le front d'eau que constitue la Meurthe et marque ainsi une profonde réorganisation de l'espace urbain.

11 Adapter l'espace urbain à la nouvelle donne économique et démographique, c'est aussi l'objectif des opérations de réhabilitation et de rénovation des grands ensembles dans les nouveaux Länder que présente Axelle de Gasperin. En effet, l'effondrement industriel de l'ex-RDA a déclenché un déclin démographique d'une ampleur inégalée, puisque la plupart des villes ont perdu jusqu'à $20 \%$ de leur population. Ainsi, cette évolution, qui a multiplié les friches industrielles, a conduit à une augmentation considérable du nombre de logements vides, en particulier dans les grands ensembles qui, dans les anciens pays communistes, représentent toujours une part très importante du bâti urbain. Le problème se pose avec une singulière acuité dans les villes nouvelles socialistes constituées en totalité de grands ensembles qui, en raison de l'insuffisante qualité du bâti, se dégradent rapidement et représentent une forme de 
logement de moins en moins prisée. L'ampleur du problème a poussé le gouvernement fédéral à intervenir dans le cadre d'un vaste programme de rénovation urbaine appelé «Stadtumbau Ost ». Il s'agit en l'occurrence, non seulement de « rétrécir » les villes par des programmes ciblés de démolition des logements vides, mais aussi de restructurer profondément les villes et de leur redonner une nouvelle image.

Dans les villes industrielles confrontées à des problèmes de reconversion, la réhabilitation des espaces dégradés, abandonnés par les activités en déclin, passe aussi souvent par la conservation d'une partie des héritages réaménagés pour accueillir de nouvelles activités ; même si seule une faible partie de ces espaces peut être concernée. C'est l'exemple des magasins d'usine installés sur d'anciens sites industriels textiles auquel s'intéresse Nicolas Lebrun. À Roubaix, où la fonction de production a disparu, les magasins d'usine se sont ainsi multipliés et la fonction commerciale a réinvesti une usine textile restaurée et presque parfaitement conservée. À Troyes par contre, où se trouve le premier site français de magasins d'usine, ceux-ci se sont installés dans des centres commerciaux périphériques, sur des sites où les héritages industriels sont quasiment inexistants. Néanmoins, ces magasins réutilisent l'image industrielle du site et c'est aussi suivant cette optique qu'a été installé récemment un centre de magasins d'usines dans le centre-ville de Roubaix. Le commerce est alors utilisé comme un instrument de réhabilitation et de renouvellement urbain.

13 La disparition du Rideau de fer en Europe a aussi entraîné la libération de nombreux terrains militaires qui sont devenus de vastes friches, souvent plus difficiles encore à réutiliser que les friches industrielles. L'exemple de la tentative de recyclage de friches aéroportuaires militaires analysé par Antoine Beyer illustre ainsi la difficulté de réutiliser des espaces conçus et implantés en fonction d'une logique totalement différente de celle à laquelle on les destinait dans le cadre du processus de reconversion. Le groupe immobilier Wiggins a en effet tenté d'exploiter l'opportunité foncière que représentait la possibilité d'acheter à très bas coût des équipements aéroportuaires de grande capacité pour créer un réseau d'aéroports destinés à accueillir les trafics provenant du délestage des grands aéroports saturés. La déconfiture finale du groupe est venue du fait qu'il a créé un réseau d'aéroports qui, pour diverses raisons, ne correspondait pas aux besoins et à la logique de fonctionnement du transport aérien civil.

14 Les quelques exemples développés dans ce numéro de la RGE montrent ainsi la richesse de ce thème de la réhabilitation des espaces industriels et urbains dégradés, qui offre à la géographie un vaste champ d'investigation. Celui-ci constitue un des axes de recherche du CERPA, le laboratoire de géographie de l'université de Nancy 2, représenté ici par quatre auteurs, dont deux jeunes chercheurs. Les trois contributions d'auteurs non nancéiens montrent que ce sujet peut constituer un de ces thèmes de recherche fédérateurs, propre à permettre les approches les plus diverses et par là même complémentaires: analyse des paysages, évaluation des politiques d'aménagement urbain et des stratégies de reconversion et de développement économique. Toutes ces approches sont présentes dans ces articles et montrent, s'il en était besoin, que la géographie est riche de sa diversité et constitue un savoir vivant en prise avec les problèmes de la société. 


\section{BIBLIOGRAPHIE}

BAUDELLE G. (1995). - Le système spatial de la mine, l'exemple du bassin houiller du Nord-Pas-de-Calais, thèse de Doctorat d'État ès Lettres, Lille III.

CHALINE C. (1999). - La régénération urbaine, Que sais-je ?, Paris, Presses Universitaires de France, $127 \mathrm{p}$.

HOLZ J.-M. (1987). - Les vieux bassins industriels : la dialectique ternaire sclérose-rupture créatricerenouveau, $112^{\mathrm{e}}$ Congrès National des Sociétés Savantes, Lyon, Géographie, p. 119-127.

\section{AUTEUR}

\section{MICHEL DESHAIES}

Université Nancy 2 - Centre d'Études et de Recherche sur les Paysages (CERPA) ; BP 33-97 - 54015 Nancy Cedex ; Tel. : 0383968421 ; michel.deshaies@univ-nancy2.fr 